\title{
Re: Ureteral Length in Live Donor Kidney Transplantation: Does Size Matter?
}

\section{Ooms LS1, Slagt IK1, Dor FJ1, Kimenai HJ1, Tran KT1, Betjes MG2, IJzermans JN1, Terkivatan T1}

\author{
1 University Medical Center Rotterdam, Erasmus MC, Department of Transplant Surgery, Department of Surgery, Rotterdam, The Netherlands \\ 2 University Medical Center Rotterdam, Erasmus MC, Department of Nephrology, Department of Internal Medicine, Rotterdam, The Netherlands
}

Transpl Int 2015;28:1326-1331. doi: 10.1111/tri.12635. Epub 2015 Aug 6.

\section{EDITORIAL COMMENT}

Ureter-related urological complications, such as urinary leakage and ureter strictures may lead to increased morbidity and prolonged hospital stay in kidney transplant recipients.

In this retrospective study, the authors have evaluated the role of ureteral length on urological complications in 198 living donor kidney recipients followed for a median of 26 months. After shortening every ureter before an intravesical or extravesical ureteroneocystostomy over an ureteral stent, ureteral lengths have been measured in all recipients and used to divide recipients into three categories as short $(\leq 8.5 \mathrm{~cm})$, medium $(8.6-10.9 \mathrm{~cm})$ and long $(\geq 11 \mathrm{~cm})$ ureters. Percutaneous nephrostomy tube (PCN) placement had been considered as the primary outcome for urological complications. Eightten percent of the recipients in the short ureter, 20\% of the medium ureter and $21 \%$ of the long ureter category had experienced PCN placement ( $p=0.886)$. Even after excluding the PCN placements for simple hydronephrosis cases, no significant difference were observed between three ureter categories. Although the number of PCN placements was higher than in the literature, the authors claim that they use a lower threshold for PCN placement even in low-grade hydronephrosis. Risk factor analysis for gender, arterial multiplicity and type of ureteroneocystostomy showed no differences in PCN placements between the three ureteral length categories. The authors have concluded that ureteral length alone did not seem to influence the number of urological complications in living donor kidney transplantation. However, further research should be planned on microvascular blood flow of the ureter to enlighten the risk factors for the development of urological complications.

\section{Re: Cancer: A "Stem-cell" disease?}

\section{Tu SM}

The University of Texas MD Anderson Cancer Center, Department of Genitourinary Medical Oncology, Texas, USA

Cancer Cell lnt 2013;13:40. doi: 10.1186/1475-2867-13-40.

\section{EDITORIAL COMMENT}

The etiopathogenesis of cancer has not been fully outlined yet. Genetic, epigenetic mechanisms and microenvironment play a major role in the etiopathogenesis of cancer. A new phrase is being used for cancer in the recent literature: "stem-ness" accounts for the dormancy, regeneration, heterogeneity, immunity and metastasis of cancer. In 1855, R. Virchow proposed that cancers arise from the activation of dormant, embryonic-like cells present in mature tissue. After this theory, Lapidot provided the first evidence to support the cancer stem cell (CSC) hypothesis when they used cell-surface protein markers to identify a relatively rare population of stem-like cells. CSCs in solid tumors have since been identifed using cell-surface protein markers in tumors of the prostate. There are many hypotheses in the literature about the relationship between cancer and stem cell. These hypotheses are focused on cancer cells arising from stem cells or progenitor cells or differentiated cells. Cancer cells and stem cells are like mirror images of each other. There appears to be malignant potential in every stem cell and stem cell potential in malignant cell. Both of them have some features as drug resistance, evading apoptosis, proto-oncogene/oncogene, immunity, tissue invasion and metastasis/morphogenesis and migration, heterogeneity/multipotentiality, active telomerase, onco-niche/stem cell niche, asymmetric divisions/aneuploidy, sustained angiogenesis, limitless self-renewal potential, and hypoxia. Many researchers belive that in the near future the characterization of CSCS will likely play a role in the development of novel targeted therapies.

Fehmi Narter, MD 